and thus to risk the neglecting of other departments of your education which are of equal consequence, and, perhaps, greater, in the ordinary practice of the profession. There can be little apology for the student of the present day not making himself familiar with all the operations in surgery; but there can be still less for him who neglects the study of those minor details which he is much more frequently required to put into useful execution. I trust that my example may bave that infuence upon you as shall encourage you to look with interest upon all departments of this branch of the profession, and that you will not fail to be impressed with the truth of the saying, that good surgery depends as much upon the minutia of practice as upon those exciting achievements which are from time to time witnessed in the operating theatre of a hospital; and, moreover, that you will always keep in view, that whatever praise may be due to a surgeon for the able perforinance of an operation, when such a proceeding is absolutely necessary, there is much more due to him who, by his skill, can avert the necessity for such an extreme measure.

In acquiring a knowledge of the principles and practice of surgery let me earuestly intreat you to devote as inuch of your time to hospital attendance as you can possibly spare from other arocatious. Bear constantly in mind that it is not alone by listening to lectures that you are to become usefully proficient. There are few subjects which any teacher can bring under your notice which will not be found treated of at greater length in published works, and the success and value of his prelections will depend chiefly upon the manner in which he brings each topic under notice, and the illustrations which he brings to bear upon it, whether these be in the shape of wellselected cases, preparations, diagrams, or examples of disease which may be open to the cognisance of all in the hospital wards. A good and plain digest of the present condition of surgery is, in my opinion, what the young student principally stands in need of, and any views peculiar to the teacher himself, which unst, of necessity, bear small proportion to the whole matters to be discussed in the course, can readily be interwoven in an appropriate manner with the ordinary lectures. But it is not so much to hear novelty that you are required to attend surgical lectures, as that you should acquire a knowledge of ordinary doctrines and ordinary modes of practice; and such knowledge is most appropriately fixed in your mind by the study of cases in the hospital. Remember that it is not to see the great cases in surgery only that you are required to attend those institutions. These are but " few and far between" in the routine of daily business, and your attention should be as sedulously given to the treatment of an ulcer or some such case as to the most important in the catalogue of surgical diseases. Indeed, it is not unusual to see a surgeon of admirable skill more puzzled with the treatment of some small and insignificant-looking sore than in the treatment of such cases as are generally esteemed of greater moment; and it is wonderful how slight a change, if judiciously adopted, will induce a successful result. These, Gentlemen, are points of prac. tice which can only be learned by that kind of personal experience which is to be had incare. fully watching the progress of cases of dis. ease: you would do well to take notes on those occasions, - for there is no better method of impressing facts on your mind; and as in your future intercourse with your professional brethren you will frequently have to enter upon explanations and details regarding diseases under your charge, the free use of your pen and thoughts in the early periods of your studies will greatly facilitate this part of youl avocations hereafter.

TUMOUR OF THE ANTRUM. REMOVAL OF THE UPPER JAW BONE.

To the Editor of THE LANCET.

Srr,-As the India medical periodicals for July last have not, as yet, perhaps reached you, and the enclosed case, which has been forwarded to me by the overland mail, origi. nally published in the "Iudia Jour. of Med. and Phys. Sciences" seems an important one, you will, I dare say, give it a place in The Lancet. It relates to the removal of the superior maxillary bone, by operation, in consequence of a tumour in the antrum. The operator was my friend, Mr. Raleigh, surgeon of the Native Hospital, and piofessor of surgery in the Medical College, Calcutta. The result of the operation proved very satisfactory, although, at one time, the symptoms of reaction threatened to be urgent, but were speedily reduced by judi. cious treatment. You will, I think, admit that, considering its formidable character, ten minutes was a short time for the operation. It is gratifying, too, to learn, from Mr. Raleigh's success, that much more of the osseous structure for the support of in portant parts, to say nothing of the preven tion of deformity, may be preserved, in such an operation, than might be anticipated, by the use of the chisel. It is no less satisfactory to think that the precaution of tying the carotid, or internal maxillary, may be safely dispensed with. I am, Sir, your obedient servant,

$$
\text { J. Grant, }
$$

Bengal Medical Establishment.

Grosvenor-place, Bath, Oct. 25, 1843.

Dunomoey, a native female, of tolerably robust frame, and of healthy general appear. 
ance, aged about twenty-seven years, mother of two children, applied at the Native Hospital for the removal of a tumour of the left side of the face, which she first perceived about seven months since, and which, during the last month, had rapidly increased in growth. At the time of applying at the hospital the disease presented, externally, a large semi-globular swelling, occupying the cheek of the affected side, from the orbit downwards.

On examination of the interior of the mouth, it was observed that a tumour, occupying the left antrum Highmorianum, protruded from, and had partially absorbed, the bony parietes of that cavity, so as to form a hard half-bony, but in some parts elastic swelling, of the outer surface of the superior maxillary bone, of the size of a half-section of a common sized billiard-ball, extending from the inferior margin of the orbit to the socket of the teeth, downwards, anteriorly to the nasal process, and posteriorly to the bulbous process of that bone; whilst, in the roof of the mouth, a corresponding bulging: of the palatine surface of the bone existed, to the exteut of half a large walnut, so as completely to occupy the left half of the palate only. On the most prominent point of the tumour externally ulceration had commenced. The whole of the teeth of that half of the upper jaw, although apparently sound, were quite loose, and partially ejected from the alveolar processes. So far as could be ascertained by external inspec. tion, the disease appeared to be confined to the left superior maxillary bone, and not to have ascended beyond the natural limit of the orbit.

June 4. The patient was placed on her back, on an operating table of ordinary height; her head, which was supported by a firm pillow, being turned to the right side, so as to afford me command over the left side of the head and face; in this position she was steadily held by my two hospital assistants, Messrs. Foy and Tresham, whilst Dr. R. Stuart, assistant-surgeon of the institution, stood on the opposite side of the patient, prepared to make pressure on the carotid artery, in case of necessity for doing so.

The left anterior incisor tooth being first extracted with a forceps, I commenced an incision over the middle of the zygomatic arch, and carried it down, cutting at once completely through the soft parts, to the angle of the mouth, avoiding the course of the parotid duct, which $I$ considered would be below, and posterior to, any part of the course of my incision; and then, by free strokes with the knife, I dissected up the muscles and integuments of the face, so as (when they where reflected on the forehead) completely to expose the external surface of the tumoui, and lay bare the superior maxillary and malar bones, in a line with the upper edge of the zygomatic arch, inferior margin of the orbit, and root of the nasal process of the superior maxillary bone. In the course of this dissection the cartilaginous septum of the nose having been separated from the spinous process, and the left ala detached from its connections, they were retracted in common with the whole cheek, by an assistant.

During this first stage of the operation, copious hæmorrhage ensued from the vessels supplying the integuments and muscles of the face, and the facial artery required compression for a time, by the thumb and finger of an assistant. Some inconvenience was experienced by large quantities of blood flowing into the fauces, and the efforts of the patient to cough it up; the loss of blood, however, in all, did not exceed sixteen or twenty ounces.

As soon as the bleeding was sufficiently restrained to admit of my pursuing the operation, $I$ introduced one blade of a strong pair of bone-forceps into the nostril, and the other into the mouth, and, with no great force, at two nips, divided the bony palate through its centre, as far back as the transverse palatine suture. I now found that by grasping the whole jaw, including the tumour, I could move it a little, and on insinuating the point of my forefinger between the globe of the eye and edge of the orbit, at the same time that I did so move it, I was satisfied that its orbitar plate retained its natural position, and that the configuration of the orbit had not been impaired ; I consequently determined to try to save the orbit entire, as well as the zygomatic arch, with the double object of preserving the natural protection of the eye, and of lessening the deformity probably consequent on an operation where the orbitar plate of the upper jaw, and the malar hone are removed. For this purpose I used a very sharp chisel, the blade of which was fully three-fourths of an inch wide, and a small wooden mallet (both of which had been prepared for the occasion); and, commencing at the root of the nasal process of the superior maxillary bone, in a line just below the inferior margin of the orbit, with a smart rap of the chisel by the mallet, I cut through the bone to the extent of the width of the chisel, and at three similar applications of that instrument completely divided the superior maxillary and inferior angle of the malar bone.

I now found the upper jaw with the tumour so much detached, that with little force I pressed it down with my left thumb and fingers, whilst, with a scalpel, I separated its posterior connections with the muscles, soft palate, \&c., and then, disconnecting it from the ossa palati, brought away the tumour entire, with the body of the upper jaw bone, leaving the velum palati, uvula, \&x., uninjured. The oozing of blood, from the internal deep-seated spongy osseous parts 
was so very trifling as to be suppressed by pressure with a sponge for a few seconds; nor did the hæmorrhage from the more superficial soft parts continue sufficiently long, nor was it of such importance as to require the application of a single ligature; a circumstance in further proof of the absence of any necessity for subjecting the patient to the formidable, and in itself sometimes fatal, operation of tying the carotid or internal maxillary arteries, as a precautionary measure to the removal of the superior maxillary bone.

The exposed parts having been carefully cleansed, a piece of fine sponge, sufficiently large to fill the excavation, was introduced, and the integuments, \&c., of the cheek were brought over it, and united by several sutures; the angle of the mouth being preserved in its natural position, a few narrow strips of adhesive plaster were applied in support of the sutures, so as to unite the edges of the incision with accuracy, and over this a cold-water dressing was kept constantly applied. The patient bore the operation with great fortitude, and although she became faint when near its completion, she soon rallied.

Epitome of Treatment.-Previously to the operation, which was performed at eight, a.m., June 4 th, the patient's bowels had been cleared by medicine, and immediately subsequent to operation no further treatment was required than a cold-water local dressing. Perfect quietude was enjoined, and an occasional teaspoonful of water was passed into the back of the throat to relieve thirst.

At four, p.m., her pulse beat 130, and at eight, p.m., 144; the skin was hot and dry, and she seemed to suffer headach.

5th, a.m. Had a little sleep in the night, and this morning appears easy; the face is swollen; pulse rapid, and skin hot, \&c. A purgative enema; cold local applications continued. P.M. Slept nearly through the whole day ; bowels twice moved by the injection. Fluid food and drink are administered by a teaspoon passed back into the throat.

7th. Febrile symptoms moderating; the discharge absorlued by the sponge having become offensive the sponge was withdrawn, and the internal surface of the wound was syringed with plain water every three hours, and a purgative enema was again administered.

8th. Swelling of face, heat of skin, and frequency of pulse continue; discharge from the wound, healthy pus. Castor oil, $\bar{\zeta}$ ss. Local applications continued.

14th. Doing well under the same course of treatment as before observed; on this day the sutures were all removed, the adhesive straps being retained.

17th. Up to this day progressed gradually, the bowels having been kept open by an occasional dose of oil, or an enema. The wound cleaned internally by syringe, and externally cold dressing continued. P.M. Had accession of fever. A mercurial purgative, followed by a dose of castor oil, was prescribed.

18th. Bowels freely moved; febrile symp. toms have abated.

19th. Had a good night, and is free from fever. Removed the straps of adhesive plaster, as, with exception of about three lines in extent at the upper edge of the incision, the external wound was healed. The internal surface of the excavation presents a smooth, healthy, granulated surface, from which a moderate quantity of healthy pus is secreted. Continue cold-water exterual dress. ing, syringe internal wound, and occasional aperients.

22nd. The external wound completely healed; she can masticate soft food with the right upper and lower jaws, and swallow it, or fluids, with perfect ease, and no inconvenience; no exfoliation of bone whatever has taken place.

July 1st. Perfectly recovered.

2nd. Discharged from the hospital, at which time the following was the condition of the parts involved in the operation :-

Internally.-The chasm occasioned by the removal of the superior maxillary bone is lined and partially filled up by granulation, having the colour and smooth appearance of the natural lining of the mouth and fauces; the soft palate and uvula are in their original state.

Externally.-A slight degree of flattening of the left cheek, and the cicatrix of the incision, are the only unnatural appearances, and it is quite extraordinary how trifling a degree of deformity exists; the patient can speak quite distinctly, but with a thickness or nasal intonation of voice.

Remarks.-In the several descriptions which I have read of this operation (and the directions given in some elementary surgical works for its performance), it appears evident that the orbitar plate of the superior maxillary bone has been removed, as well as the entire malar bone also, a circumstance which must of necessity have rendered the operation tedious, as well as very much added to the subsequent deformity, and exposure of the globe of the eye, beyond that which is incurred by the mode of operating which I pursued in the foregoing case, and which enabled me, with perfect deliberation, to complete the operation within ten minutes (timed by a watch by Mr. Kean, Ceylon student of the Medical College), saving the lateral bony arch of the nose, orbit, and the zygomatic arch, and thus preserving the prominent formation of the cheek, and the natural protection of the eye against external violence, objects which $I$ should recommend being always aimed at where the parts are not so directly involved in disease as to re. quire their removal on that account. 
I wish distinctly to explain that the sharp head hot; drivelling; bowels confined and edge of the chisel, smartly propelled by the evacuations light in colour; skin cold, the mallet, produced a clean division of the arms and legs presenting a blue and conbones, without occasioning any splintering, gested appearance. It was evident to me and that the entire separation of the upper that these symptoms were identical with bony connection of the superior maxillary bone did not occupy more than one minute of time; whereas, in dividing these parts in the usually advised manner, with a saw or forceps, separating the inferior muscles, \&c., of the eye, from the orbitar plate, and wrenching that process from its junction by suture with surrounding bones within the orbit, I can easily conceive much time must be expended to the excruciating agony of the patient, and in such instances as exemplified by the present case, to the unnecessary destruction of important parts, and uncalled for extent of eventual deformity. It will be noticed that $I$ did not divide the centre of the lip, as is commonly recommended, and I found no difficulty in completely exposing the bony side of the face by reflecting the soft parts from the lateral incision.

Walter Raleigh,

Surgeon of the Native Hospital. Calcutta, July 4, 1843 .

\section{STRIDULOUS CONVULSION IN INFANTS.}

\section{To the Editor of ThE LANCET.}

Sir, - I transmit, for insertion in THE LANCET, a case of stridulous convulsion in an infant, corresponding in results with the very original and scientific details of the one published by Dr. Marshall Hall in your

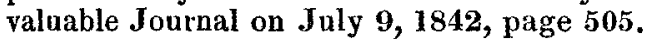

The disease in question is considered by Dr. M. Hall to consist in a peculiar susceptibility of the excito-motor property of the nervous system, the immediate attacks being the action of sources of irritation or excitement on that property, the susceptibility of which should, therefore, if possible, be diminished, if the causes of excitement cannot be carefully avoided; the most obvious of which are dentition, indigestible food, morbid alvine matters, external agents, and mental emotions. Fully convinced of the rationality of Dr. Hall's remarks, $I$ think the following case is illustrative of the soundness of his conclusions. In January last I was summoned to the son of a gentleman in this town, xtat. three months, who had been attacked with frequent fits of croupal inspiration, which passed away in a few minutes, the child afterwards appearing as well as usual. The mother expressed great anxiety for him, as her eldest son had died during infancy with symptoms which began in the same way. In a few days these attacks were accompanied with severe convulsions, some of which lasted eight or ten hours. The patient was stout and remarkably well-formed; gums broad and inflamed; those of the elder son, who was under my care. The same cause, probably, existed in both instances, viz., dentition, producing a peculiar susceptibility of the excito-notor property of the nervous system.

I lanced the gums all round twice a-day; leeched the temples twice or thrice; clothed the child in flannel from the neck to the wrists and ankles; Ikept the head constantly cool with a lotion of spirits of wine and water; had the feet frequently immersed in a foot-bath of warm water with mustard, and the apartment darkened. That the milk of the mother might not be a source of irritation, a healthy young wet-nurse was procured, and allowed to nurse the infant ouly once in three hours. To remove morbid alvine evacuations, a grain of calomel and five grains of rhubarb, were given every night. At this time the patient had six or eight fits of convulsions daily; at the end of the first week, two or three fits in the twenty. four hours; in three weeks from the commencement the convulsions were less frequent and less severe; the gums not so inflamed; the blueness of the arms and feet less; the head cooler; the countenance more tranquil; the stools healthier; and the sleep often sound and refreshing.

Feb. 20. The gums lanced once a-day, and this was repeated until June, during which time the croupal inspiration was seldom heard, and there were only three attacks of convulsions. The incisors came through the gums one by one. About the middle of the month the child to be carried into the open air every day, if the wind be not from the north or north-east. A powder every third night. The gums were now lanced twice a-week. At the latter end of the month, upon one occasion, they were not lanced on the usual day, and on the following morning the convulsions returned with great violence. They soon, however, yielded to a free division of the gums.

July 1. The gum covering the canine tooth on the right side in the upper jaw became inflamed, a slough formed, and a conical shell, resembling the enamel of a canine tooth, came away. Four bicuspid teeth visible through the gums; at the end of July they came through. An entire absence of convulsions aud croupal inspiration for several weeks. The weather being hot, the flannel coverings are removed. He now takes a small quantity of food through a bottle twice a-day, preparatory to weaning. $\mathrm{He}$ grows, and is fleshy and healthful in appearance. If the gums be not scarified on the regular day, he often becomes restless and feverish, and the bowels are relaxed. August. Hooping-cough being epidemic, 